\title{
Magnetothermopower and Nernst effect in unconventional charge density waves
}

\author{
Balázs Dóra ${ }^{1}$, Kazumi Maki², András Ványolos ${ }^{3}$ and Attila Virosztek ${ }^{3,4}$ \\ 1 The Abdus Salam ICTP, Strada Costiera 11, I-34014, Trieste, Italy \\ 2 Department of Physics and Astronomy, University of Southern California, Los Angeles CA 90089-0484, USA \\ ${ }^{3}$ Department of Physics, Budapest University of Technology and Economics, H-1521 Budapest, Hungary and \\ ${ }^{4}$ Research Institute for Solid State Physics and Optics, P.O. Box 49, H-1525 Budapest, Hungary
}

(Dated: November 12, 2018)

\begin{abstract}
Recently we have shown that the striking angular dependent magnetoresistance in the low temperature phase (LTP) of $\alpha$-(BEDT-TTF $)_{2} \mathrm{KHg}(\mathrm{SCN})_{4}$ is consistently described in terms of unconventional charge density wave (UCDW). Here we investigate theoretically the thermoelectric power and the Nernst effect in UDW. The present results account consistently for the recent data of magnetothermopower in $\alpha$-(BEDT-TTF $)_{2} \mathrm{KHg}(\mathrm{SCN})_{4}$ obtained by Choi et al. (Phys. Rev. B, 65, 205119 (2002)). This confirms further our identification of LTP in this salt as UCDW. We propose also that the Nernst effect provides a clear signature of UDW.
\end{abstract}

PACS numbers: 75.30.Fv, 71.45.Lr, 72.15.Eb, 72.15.Nj

Recently many possible candidates for unconventional charge density wave (UCDW) and unconventional spin density wave (USDW) have been proposed, though in most cases definitive confirmation is still lacking. These are the antiferromagnetic phase of $\mathrm{URu}_{2} \mathrm{Si}_{2}$ 1, 2], the pseudogap phase in high $T_{c}$ cuprates [3, 4, [5, 6], the $\mathrm{CDW}$ in $\mathrm{NbSe}_{2}$ 7, 8 ] and the low temperature phase (LTP) in $\alpha$-(BEDT-TTF $)_{2} \mathrm{MHg}(\mathrm{SCN})_{4}$ with $\mathrm{M}=\mathrm{K}, \mathrm{Rb}$ and Tl $9,10,11,12,13]$. In the last system not only the qualitative features of LTP, like the absence of a clear charge order, but also both the temperature and magnetic field dependence of the threshold electric field [9, 10, 11] and the striking angular dependent magnetoresistance (ADMR) 12, 13] are fully consistent with UCDW. In these studies the quantization of the quasiparticle spectrum in the presence of magnetic field as considered by Nersesyan et al. [14, 15] plays the crucial role.

The object of the present paper is to extend our earlier study to the thermoelectric power and Nernst effect in UDW (i.e. UCDW and USDW) in the presence of magnetic field. When the Zeeman splitting or the Pauli term due to magnetic field is negligible compared to the orbital effect, there will be no distinction between UCDW and USDW, which we will assume in the followings. First we discuss briefly how the effect of magnetic field is incorporated following Refs. [14, 15]. Then we construct the expressions for thermopower and Nernst effect in UDW. These are compared with a recent data by Choi et al. 16] on $\alpha$-(BEDT-TTF $)_{2} \mathrm{KHg}(\mathrm{SCN})_{4}$. Indeed we can describe the experimental data very consistently.

In the absence of magnetic field the quasiparticle energy in UCDW is given by 17 ]

$$
(E+\varepsilon(\mathbf{k})) \Psi=\left(-i v_{a} \partial_{x} \rho_{3}+\Delta \cos \left(c k_{z}\right) \rho_{1}\right) \Psi,
$$

where $\rho_{i}$ 's are the Pauli matrices acting on spinor space of the left and right moving electrons on the quasi-one dimensional Fermi surface and the imperfect nesting term $\varepsilon(\mathbf{k})$ is given by $\underline{13}$

$$
\varepsilon(\mathbf{k})=\sum_{n=-\infty}^{\infty} \varepsilon_{n} \cos \left(2 \mathbf{b}_{n}^{\prime} \mathbf{k}\right)
$$

where $\mathbf{b}_{n}^{\prime}=b^{\prime}\left[\hat{\mathbf{r}}_{b}+\tan \left(\theta_{n}\right)\left(\hat{\mathbf{r}}_{a} \cos \phi_{0}+\hat{\mathbf{r}}_{c} \sin \phi_{0}\right)\right], \varepsilon_{n}=$ $\varepsilon_{0} 2^{-|n|}, \tan \left(\theta_{n}\right)=\tan \left(\theta_{0}\right)+n d_{0}, \tan \left(\theta_{0}\right) \simeq 0.5, d_{0} \simeq$ $\left.1.25, \phi_{0} \simeq 27^{\circ} 18,19,20\right]$, and $\phi$ is the angle the projected magnetic field on the $a-c$ plane makes from the $c$-axis. This generalized imperfect nesting term arises from an effective tight binding approximation, where hopping takes place between sites in the $\hat{\mathbf{r}}_{b}$ direction and along nearest neighbour chains oriented in the $\hat{\mathbf{r}}_{a} \cos \phi_{0}+\hat{\mathbf{r}}_{c} \sin \phi_{0}$ direction. Eq. (10) is readily solved as

$$
E= \pm \sqrt{\left(v_{a} k_{x}\right)^{2}+\Delta^{2} \cos ^{2}\left(c k_{z}\right)}-\varepsilon(\mathbf{k}),
$$

where $k_{x}, k_{z}$ are wavevector components parallel to the $a$ and $c$ axis in $\alpha$-(BEDT-TTF $)_{2} \mathrm{KHg}(\mathrm{SCN})_{4}$ salt. We note here that imperfect nesting breaks the particle-hole symmetry in general. In the presence of magnetic field Eq. (11) is transformed as

$$
E \Psi=\left(-i v_{a} \partial_{x} \rho_{3}+\Delta c e B x \cos (\theta) \rho_{1}\right) \Psi,
$$

where for the moment we ignored the imperfect nesting term. $\theta$ is the angle the magnetic field makes from the $b^{*}$ axis. We define $b^{*}$ as the direction perpendicular to the $a-c$ plane. Eq. (4) is readily solved as 14, 15]

$$
E^{2}=2 n v_{a} \Delta c e|B \cos \theta|,
$$

where $n=0,1,2 \ldots$. We note that Eq. (44) is the same as the Dirac equation in a constant magnetic field and has been studied since 1936[21]. The Landau wavefunctions are given by

$$
\begin{gathered}
\Psi_{0}=\left(\begin{array}{c}
i \\
1
\end{array}\right) \phi_{0}, \\
\Psi_{n \neq 0}=\frac{1}{\sqrt{2}}\left[\left(\begin{array}{c}
1 \\
i
\end{array}\right) \phi_{n-1} \pm\left(\begin{array}{c}
i \\
1
\end{array}\right) \phi_{n}\right],
\end{gathered}
$$


where $\phi_{n}$ is the $n$-th wavefunction of a linear harmonic oscillator with parameters "mass" $m=1 / 2 v_{a}^{2}$ and "frequency" $\omega=2 v_{a} \Delta c e B \cos (\theta)$. From Eq. (7) it is obvious, that the $n \neq 0$ levels are twofold degenerate, since $\Psi_{n \neq 0}$ is composed of the $n-1$-th and $n$-th wavefunction of the harmonic oscillator. Now making use of the Landau wave functions we evaluate the contribution from the imperfect nesting term as perturbation. Then we get for the Landau levels:

$$
\begin{gathered}
E_{0,1}=-E_{0}^{(1)}, \\
E_{1,1}= \pm E_{1}-E_{1}^{(1)}, \\
E_{1,2}= \pm E_{1}-E_{1}^{(2)},
\end{gathered}
$$

and

$$
\begin{gathered}
E_{n}=\sqrt{2 n v_{a} \Delta c e B|\cos (\theta)|}, \\
E_{0}^{(1)}=E_{1}^{(1)}=\sum_{m} \varepsilon_{m} \exp \left(-y_{m}\right), \\
E_{1}^{(2)}=\sum_{m} \varepsilon_{m}\left(1-2 y_{m}\right) \exp \left(-y_{m}\right),
\end{gathered}
$$

where $y_{m}=v_{a}{b^{\prime 2}}^{2}|B \cos (\theta)|\left(\tan (\theta) \cos \left(\phi-\phi_{o}\right)-\right.$ $\left.\tan \left(\theta_{m}\right)\right)^{2} / \Delta c$. We note that the imperfect nesting terms splits the $n=1$ Landau level into 2 nondegenerate levels. Also as is the case in the absence of magnetic field, the imperfect nesting term breaks the particle-hole symmetry. This particle-hole symmetry breaking is crucial in the thermoelectric power. Then keeping just the $n=0$ and $n=1$ Landau levels, the ADMR is constructed as

$$
\begin{gathered}
R(B, \theta, \phi)^{-1}=\sigma_{1}\left(\frac{\exp \left(-x_{1}\right)+\cosh \left(\zeta_{0}\right)}{\cosh \left(x_{1}\right)+\cosh \left(\zeta_{0}\right)}+\right. \\
\left.+\frac{\exp \left(-x_{1}\right)+\cosh \left(\zeta_{1}\right)}{\cosh \left(x_{1}\right)+\cosh \left(\zeta_{1}\right)}\right)+\sigma_{2},
\end{gathered}
$$

where $x_{1}=\beta E_{1}, \zeta_{0}=\beta E_{1}^{(1)}, \zeta_{1}=\beta E_{1}^{(2)}$ and $1 / \beta=$ $k_{B} T$. Here $\sigma_{1}$ is the conductivity of the $n=1$ Landau level and the contribution from the $n=0$ Landau level, $\sigma_{0}$ (which was found to be constant within the present approximation) is considered together with the conductivity of the quasi-two dimensional Fermi surface in $\sigma_{2}$, which is also assumed to be independent of $B$. Also from our construction of ADMR, Eq. (14) should work better for smaller $T$ and larger $B$. As was shown in Ref. 12, 13], Eq. (14) gives an excellent description of ADMR found in $\alpha$-(BEDT-TTF $)_{2} \mathrm{KHg}(\mathrm{SCN})_{4}$.

The diagonal component of the magnetic field dependent thermoelectric power (TEP) is also of particular in- terest. It is obtained similarly as Eq. (14), and reads:

$$
\begin{gathered}
S(B, \theta, \phi)=-\frac{R(B, \theta, \phi) k_{B}}{e}\left[\sigma_{0} \zeta_{0}+\right. \\
+\sigma_{1}\left(\zeta_{0} \frac{\exp \left(-x_{1}\right)+\cosh \left(\zeta_{0}\right)}{\cosh \left(x_{1}\right)+\cosh \left(\zeta_{0}\right)}+\zeta_{1} \frac{\exp \left(-x_{1}\right)+\cosh \left(\zeta_{1}\right)}{\cosh \left(x_{1}\right)+\cosh \left(\zeta_{1}\right)}+\right. \\
\left.\left.+x_{1}\left(\frac{\sinh \left(\zeta_{0}\right)}{\cosh \left(x_{1}\right)+\cosh \left(\zeta_{0}\right)}+\frac{\sinh \left(\zeta_{1}\right)}{\cosh \left(x_{1}\right)+\cosh \left(\zeta_{1}\right)}\right)\right)\right]
\end{gathered}
$$

We note here that $S(B, \theta, \phi)$ vanishes in the absence of imperfect nesting. Before comparing Eq. (15) with experimental data, we shall consider the Nernst effect.

The Nernst effect is the off diagonal component of the thermoelectric power in the presence of magnetic field. Also its formulation is different from above. We have seen already that quasiparticle in UDW orbits around the magnetic field. Then when an electric field $\mathbf{E}$ is applied with a perpendicular component to the magnetic field $\mathbf{B}$, the quasiparticle orbit drifts with $\mathbf{v}_{D}=(\mathbf{E} \times \mathbf{B}) / B^{2}$. Then the heat current parallel to $\mathbf{v}_{D}$ is given by $\mathbf{J}_{h}=$ $T S \mathbf{v}_{D}$, where $S$ is the entropy associated with the circling quasiparticles:

$$
S=e B \sum_{n}\left[\ln \left(1+\exp \left(-\beta E_{n}\right)\right)+\beta E_{n}\left(1+\exp \left(\beta E_{n}\right)\right)^{-1}\right],
$$

the sum over $E_{n}$ has to be taken over all the Landau levels, and the magnetic field is assumed to be perpendicular to the $a-c$ plane $\left(\theta=0^{\circ}\right)$. Then for small $T$ and large $B$, Eq. (16) is well approximated by taking the $n=0$ and $n=1$ Landau levels. Moreover, when the zeroth order contribution from the energy spectrum (i.e. the Landau levels without imperfect nesting) is finite, we can neglect higher order terms, namely the effect of imperfect nesting by setting $\zeta_{0}=\zeta_{1}=0$. With this simplification, the entropy reads as

$$
S=2 e B\left[\ln (2)+2 \ln \left(2 \cosh \left(\frac{x_{1}}{2}\right)\right)-x_{1} \tanh \left(\frac{x_{1}}{2}\right)\right] .
$$

So the Nernst coefficient in this configuration can be calculated, after considering the effect of the two dimensional parts of the Fermi surface:

$$
\begin{gathered}
S_{x y}=\alpha_{x y}=-\frac{S}{B \sigma}=\frac{1}{\sigma}\left[\frac{L_{2 \mathrm{D}}}{1+\gamma^{2} B^{2}}-\right. \\
\left.-2 e\left(\ln (2)+2 \ln \left(2 \cosh \left(\frac{x_{1}}{2}\right)\right)-x_{1} \tanh \left(\frac{x_{1}}{2}\right)\right)\right],
\end{gathered}
$$

where $\sigma=1 / R=4 \sigma_{1} /\left(\exp \left(x_{1}\right)+1\right)+\sigma_{2}$ from Eq. (14), $L_{2 \mathrm{D}}$ stems from the two dimensional cylinders of the Fermi surface, $\gamma=e \tau / m, \tau$ is the field-free relaxation time at the Fermi level 22, 23], $m$ is the effective mass of the electron.

In Figs. 11 and 2] we compare Eq. (15) to the experimental data of the diagonal thermopower (Seebeck 
coefficient) in a magnetic field perpendicular to the conducting plane $\left(\theta=0^{\circ}\right)$ for heat current applied in the $a$ and $c$ directions, respectively. As is readily seen we can have excellent fittings. From these fittings we can deduce $\Delta \sim 17 \mathrm{~K}, v_{a} \sim 10^{6} \mathrm{~cm} / \mathrm{s}$, which are very consistent with earlier results. As to $\varepsilon_{0}$, its value is obtained as $9 \mathrm{~K}$ for $T=1.4 \mathrm{~K}$, which is of the same order of magnitude as what we obtained previously in Ref. [13], but for higher temperatures (above half $T_{c}$ ) we obtain $\varepsilon_{0}$ around $40 \mathrm{~K}$. This bigger value might stem from the neglect of the effect of higher Landau levels (which becomes more important as the temperature increases), and also of the magnetic field and temperature dependence of $\sigma_{1}$ and $\sigma_{2}$. In Fig. 3 we compare our theoretical results to the experimental data on Nernst effect. From this we can deduce the same parameters, and the new fitting parameter, $\tau=10^{-11} \mathrm{~s}$, assuming $m$ to be twice the free electron mass. This can be converted to temperatures as $\tau^{-1} \rightarrow 4 \mathrm{~K}$, which is reasonable concerning the presence of de Haas van Alphen oscillations at $B>10 \mathrm{~T}$ at $T=1.4 \mathrm{~K}$. Finally in Fig. 团 the temperature dependence of the Seebeck coefficient is fitted at $B=12 \mathrm{~T}$. Here we assumed $\Delta(T) / \Delta(0)=\sqrt{1-\left(T / T_{c}\right)^{3}}$, which is very close to our weak coupling solution 24 . The extracted fitting parameters are again in the same order of magnitude as earlier.

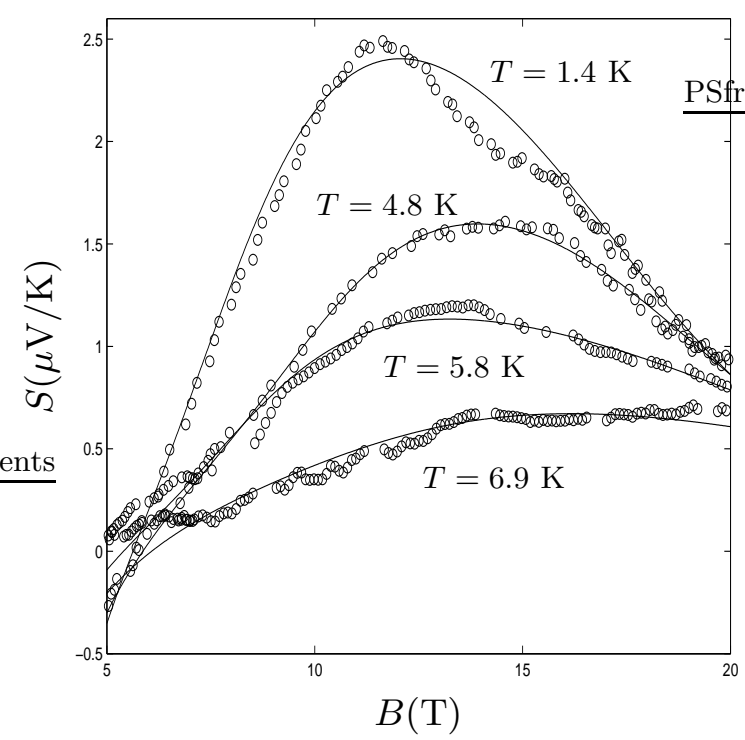

FIG. 1: The magnetothermopower for heat current along the $a$ direction is shown for $T=1.4 \mathrm{~K}, T=4.8 \mathrm{~K}, T=5.8 \mathrm{~K}$ and $T=6.9 \mathrm{~K}$ from top to bottom, the circles denote the experimental data from Ref. [16], the solid line is our fit based on Eq. (15).

As we have shown the quasiparticle spectrum as obtained can describe the magnetothermopower as observed in LTP in $\alpha$-(BEDT-TTF $)_{2} \mathrm{KHg}(\mathrm{SCN})_{4}$. Together with the earlier results on ADMR, the present work further confirms our proposition that LTP in $\alpha$-(BEDT$\mathrm{TTF})_{2} \mathrm{KHg}(\mathrm{SCN})_{4}$ salt is UCDW. Also the Nernst ef-
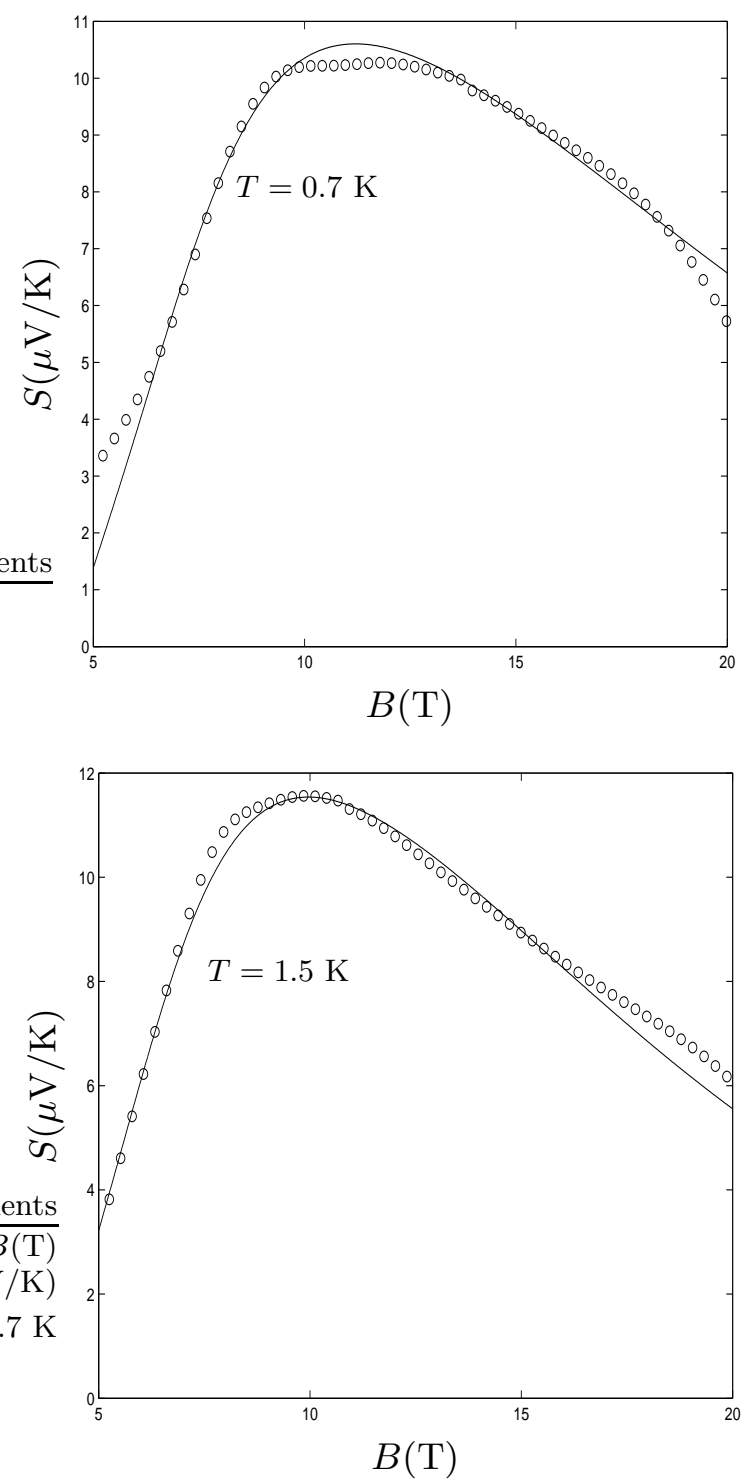

FIG. 2: The magnetothermopower for heat current along the $c$ direction is shown for $T=0.7 \mathrm{~K}$ (upper panel) and $T=1.5 \mathrm{~K}$ (lower panel), the circles denote the experimental data from Ref. [16], the solid line is our fit based on Eq. (15).

fect we obtained is rather large and independent of the imperfect nesting term. Therefore we may consider the Nernst effect as the hallmark of UDW. No corresponding term exists in conventional DW. The $B$ dependence of the Nernst effect is very similar to the one obtained in the vortex state of dirty type II s-wave superconductors 25, 26, 27, 28]. It has been claimed by Wang et al. [29], that the large Nernst and Ettinghausen effect in the pseudogap phase is the signature of the presence of superconducting vortices. But the present results point clearly to the alternative possibility. Indeed the beautiful experimental data from underdoped LSCO appear to indicate that the pseudogap phase is UDW as proposed by many people $[\underline{3}, 4,[5,[6]$. Of course the quantitative comparison between experiment and theory as done 


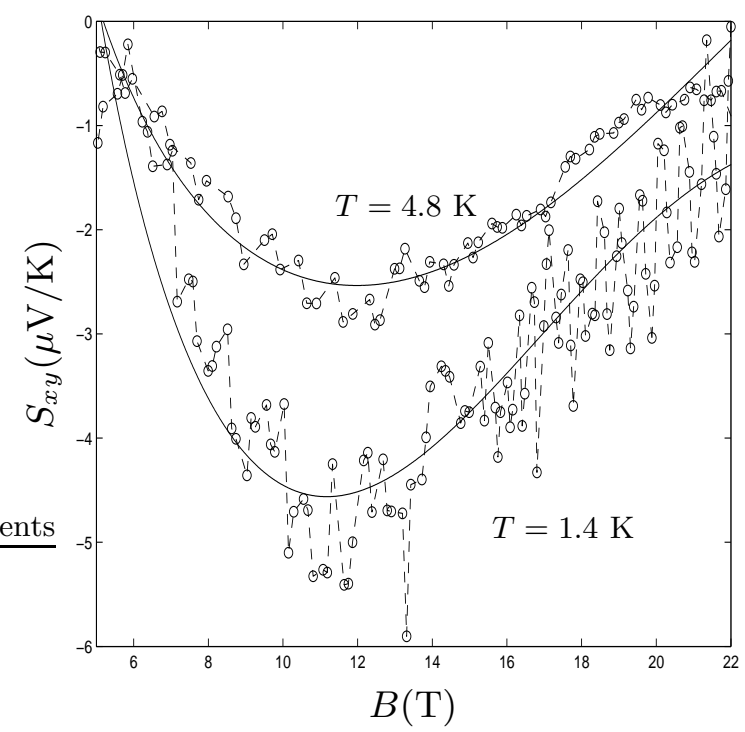

FIG. 3: The Nernst signal for heat current along the $a$ direction is shown for $T=1.4 \mathrm{~K}$ and $T=4.8 \mathrm{~K}$ (from bottom to top), the dashed lines with circles denote the experimental data from Ref. [16], the solid line is our fit based on Eq. [18.

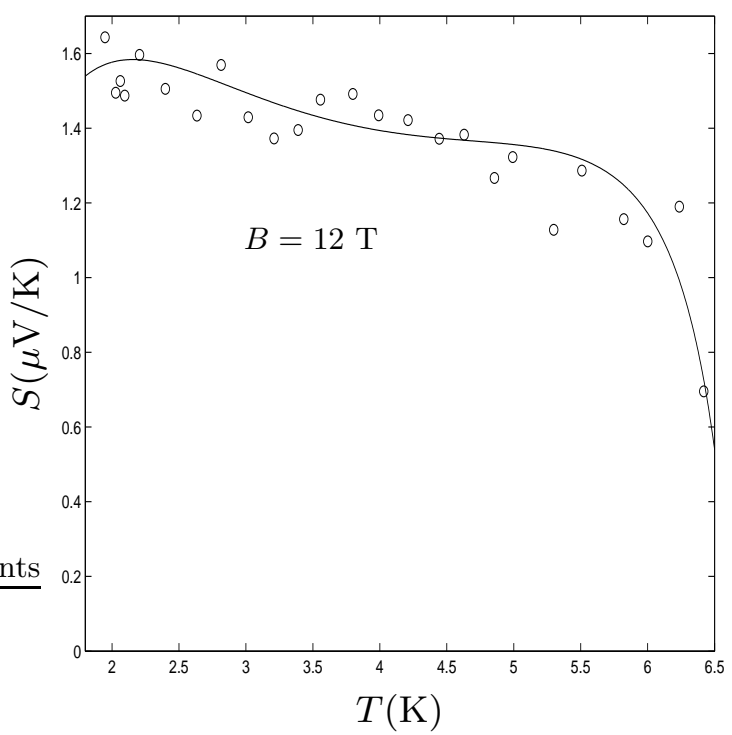

FIG. 4: The temperature dependence of the magnetothermopower for heat current along the $a$ direction is shown for $B=12 \mathrm{~T}$, the circles denote the experimental data from Ref. [16], the solid line is our fit based on Eq. (15).

here for LTP in $\alpha$-(BEDT-TTF $)_{2} \mathrm{KHg}(\mathrm{SCN})_{4}$ is highly desirable. We also believe that measurements of Nernst and/or Ettinghausen effect will prove to be decisive in other possible UDW candidate materials.

We would like to thank Eun Sang Choi for sending us the data of Ref. [16]. We have benefited from discussions with Mario Basletić, Mark Kartsovnik, Bojana Korin-Hamzić, Amir Hamzić, Silvia Tomić and Peter Thalmeier. We thank Yuji Matsuda for drawing our attention to Ref. 8]. Two of us (K.M. and B.D.) acknowl- edge the hospitality and support of the Max Planck Institute for the Physics of Complex Systems and Max Planck Institute for Chemical Physics of Solids, Dresden, where part of this work was done. This work was supported by the Hungarian National Research Fund under grant numbers OTKA T032162 and NDF45172.

[1] H. Ikeda and Y. Ohashi, Phys. Rev. Lett. 81, 3723 (1998).

[2] A. Virosztek, K. Maki, and B. Dóra, Int. J. Mod. Phys. B 16, 1667 (2002).

[3] L. Benfatto, S. Caprara, and C. Di Castro, Eur. Phys. J. B 17, 95 (2000).

[4] C. Nayak, Phys. Rev. B 62, 4880 (2000).

[5] S. Chakravarty, R. B. Laughlin, D. K. Morr, and C. Nayak, Phys. Rev. B 63, 094503 (2001).

[6] B. Dóra, A. Virosztek, and K. Maki, Acta Physica Polonica B 34, 571 (2003).

[7] A. H. Castro-Neto, Phys. Rev. Lett. 86, 4382 (2001).

[8] R. Bel, K. Behnia, and H. Berger, cond-mat/0302259.

[9] M. Basletić, B. Korin-Hamzić, M. V. Kartsovnik, and H. Müller, Synth. Met. 120, 1021 (2001).

[10] B. Dóra, A. Virosztek, and K. Maki, Phys. Rev. B 64, 041101(R) (2001).

[11] B. Dóra, A. Virosztek, and K. Maki, Phys. Rev. B 65, 155119 (2002).

[12] B. Dóra, K. Maki, B. Korin-Hamzić, M. Basletić, A. Virosztek, M. V. Kartsovnik, and H. Müller, Europhys. Lett. 60, 737 (2002).

[13] K. Maki, B. Dóra, M. V. Kartsovnik, A. Virosztek, B. Korin-Hamzić, and M. Basletić, Phys. Rev. Lett. 90, 256402 (2003).

[14] A. A. Nersesyan and G. E. Vachnadze, J. Low Temp. Phys. 77, 293 (1989).

[15] A. A. Nersesyan, G. I. Japaridze, and I. G. Kimeridze, J. Phys. Cond. Mat. 3, 3353 (1991).

[16] E. S. Choi, J. S. Brooks, and J. S. Qualls, Phys. Rev. B 65, 205119 (2002).

[17] K. Maki, Phys. Rev. B 33, 4826 (1986).

[18] A. E. Kovalev, M. V. Kartsovnik, R. P. Shibaeva, L. P. Rozenberg, I. F. Schegolev, and N. D. Kushch, Solid State Commun. 89, 575 (1994).

[19] T. Sasaki and N. Toyota, Phys. Rev. B 49, 10120 (1994).

[20] N. Hanasaki, S. Kagoshima, N. Miura, and G. Saito, J. Phys. Soc. Japan 65, 1010 (1996).

[21] W. Heisenberg and H. Euler, Z. Phys. 98, 714 (1936).

[22] S. Lambrecht and M. Ausloos, Phys. Rev. B 53, 14047 (1996).

[23] D. V. Livanov, Phys. Rev. B 60, 13439 (1999).

[24] B. Dóra and A. Virosztek, Eur. Phys. J. B 22, 167 (2001).

[25] C. Caroli and K. Maki, Phys. Rev. 164, 591 (1967).

[26] K. Maki, Phys. Rev. Lett. 21, 1755 (1968).

[27] A. Houghton and K. Maki, Phys. Rev. B 3, 1625 (1971).

[28] C.-R. Hu, Phys. Rev. B 13, 4780 (1976).

[29] Y. Wang, N. P. Ong, Z. A. Xu, T. Kakeshita, S. Uchida, D. A. Bonn, R. Liang, and W. N. Hardy, Phys. Rev. Lett. 88, 257003 (2002). 